\title{
Mecánica respiratoria en anestesia general: Revisión de conceptos
}

\section{Respiratory mechanics in general anesthesia: Concepts review}

\author{
Alejandro Placenti ${ }^{1, *}$, Matías Ramos ${ }^{1}$, Franco Fratebianchi ${ }^{1}$ \\ 1 División de Anestesia, Analgesia y Reanimación, Hospital de Clínicas "José de San Martín”, Universidad de Buenos Aires. Ciudad \\ Autónoma de Buenos Aires, Argentina.
}

Declaración de financiamiento: Este trabajo no recibió ninguna subvención específica de agencias de financiamiento público, comercial o privado. Fue realizado con el financiamiento de los autores.

Conflicto de intereses: Ninguno.

Fecha de recepción: 11 de julio de 2021 / Fecha de aceptación: 24 de octubre de 2021

\begin{abstract}
In anesthesia practice, mechanical ventilation is a fundamental tool, and its correct configuration is essential in the patients care. Airway pressure is often assumed to reflect the forces applied to the lung and is used to monitor mechanical ventilation. This assumption is erroneous because pressure acts on the respiratory system as a whole and the impact on its components will depend on the ratio of lung and respiratory system elastances. In turn, patients' lungs with the same body size and ventilated with the same tidal volume, may be subjected to different forces depending on their functional size. This is expressed under the concepts of stress and strain. Its surrogate owners, the Paw plateau and the Vt, have shown a poor correlation compared to transpulmonary pressure and the airway driving pressure. This review aims to provide the theoretical-practical tools necessary to optimize mechanical ventilation for each patient.
\end{abstract}

Key words: Driving pressure, strain/mechanical ventilation, respiratory mechanics, plateau pressure, transpulmonary pressure, elastance, stress, strain.

\section{RESUMEN}

En la práctica anestésica, la ventilación mecánica es una herramienta fundamental, y su correcta configuración es esencial en el cuidado de los pacientes. La presión de la vía aérea es, muchas veces, asumida como el reflejo de las fuerzas aplicadas en el pulmón y es utilizada para monitorizar la ventilación mecánica. Esta asunción es errónea porque la presión actúa sobre el sistema respiratorio en su totalidad y la repercusión sobre sus componentes va a depender de la relación de elastancias del pulmón y el sistema respiratorio. A su vez, los pulmones de pacientes con el mismo tamaño corporal y ventilados con el mismo volumen corriente, pueden estar sujetos a diferentes fuerzas dependiendo de su tamaño funcional. Esto es expresado bajo los conceptos de stress y strain. Sus respectivos subrogantes, $P_{\text {aw plateau }}$ y el $V t$, han demostrado tener una pobre correlación en comparación con la presión transpulmonar y la airway driving pressure. Esta revisión pretende brindar las herramientas teórico-prácticas necesarias para optimizar la ventilación mecánica para cada paciente.

Palabras clave: Ventilación mecánica, mecánica respiratoria, presión transpulmonar, elastancia, stress. 


\section{Introducción}

E n la práctica anestésica, la ventilación mecánica es una herramienta fundamental ya que es mandatoria en muchos procedimientos quirúrgicos[1]. La correcta configuración del respirador es un aspecto esencial en el cuidado de los pacientes dado que puede causar daño pulmonar significativo, un proceso conocido como Ventilator-Induced Lung Injury (VILI)[2]. Varios parámetros son determinantes de VILI como ser el stress inspiratorio, el strain dinámico y estático y la driving pressure[3].

Durante las últimas décadas hemos asistido a un cambio de paradigma en el papel de la ventilación mecánica: Desde una intervención que salva vidas, proporcionando una adecuada oxigenación a través de grandes volúmenes corrientes, hasta un contribuyente y amplificador del daño pulmonar[4]. En este escenario, la mecánica respiratoria ha ganado un rol fundamental a la cabecera del paciente. Primeramente porque muchas de estas variables o parámetros son utilizados para titular una ventilación menos injuriosa (ej. protectora). Y segundo, debido a que muchas de estas variables demostraron ser predictoras de mortalidad en el Síndrome de dificultad respiratoria aguda (SDRA) (ej. Presión meseta, Driving pressure, etc)[4] y de morbilidad en el postoperatorio[5]. Por ende, una comprensión más profunda de la mecánica respiratoria es fundamental para el anestesiólogo, con el fin de definir la configuración ventilatoria según las características particulares de cada paciente[1].

En este artículo se revisan los conceptos de presión de la vía aérea, elastancia, complacencia y resistencia del sistema respiratorio, desde un enfoque aplicable a la ventilación mecánica intraoperatoria. También se explican los términos de strain, stress y stress index. De cada tópico se agregan apartados de utilidad clínica con el fin de brindar las herramientas teóricoprácticas necesarias para interpretar la mecánica respiratoria a la cabecera del paciente.

\section{Desarrollo}

\section{Ecuación del movimiento del aparato respiratorio}

El modelo comúnmente utilizado para representar la mecánica respiratoria es el de un tubo rígido conectado a un cuerpo elástico (globo) conocido como modelo monocompartimental del sistema respiratorio[6]. Por medio de una diferencia de presión se genera un flujo de aire que produce el cambio de volumen en el cuerpo elástico[7]:

Paw $=\operatorname{Ers} \times \Delta V+\operatorname{Rrs} \times \mathrm{Vi}+\mathrm{PEEP}_{\text {tot }}$

Donde Paw es la presión generada por el ventilador, Ers es la elastancia del sistema respiratorio, $\Delta \mathrm{V}$ es el volumen insuflado por encima del volumen de final de la espiración (EELV) y corresponde al Volumen corriente o tidal, $R$ es la resistencia del sistema respiratorio, Vi es el flujo inspiratorio, y PEEPtot es la suma de la PEEP programada más la PEEP intrínseca (de existir).

La ecuación del movimiento se puede aplicar cuando se utilizan modelos de un solo compartimiento del sistema respiratorio (es decir, tubo-globo) para describir la relación dinámica entre presión, flujo y volumen[8].

En este modelo, presión, flujo y volumen son variables en función del tiempo, mientras que elastancia y la resistencia son los parámetros del modelo y contribuyen a la carga contra la que el ventilador deberá realizar el trabajo. La resistencia es la razón o ratio entre un cambio de presión y un cambio de flujo y, la elastancia es definida como la razón o ratio entre un cambio de presión y un cambio de volumen[6] (Figura 1).

La ecuación de movimiento puede entenderse como la suma de diferentes tipos de "cargas" contra las que el ventilador se enfrenta al entregar el volumen corriente al paciente y la forma en la que lo hace es por medio de la generación de presión.

Paw $=$ carga elástica + carga resistiva + carga PEEP

La carga elástica será la presión generada por el ventilador para sobrepasar las fuerzas elásticas del sistema respiratorio al entregar un volumen. La carga resistiva será la presión necesaria para vencer la resistencia al entregar un flujo inspiratorio. La carga PEEP será la presión que genera la distensión estática y basal del sistema respiratorio[7].

Por lo tanto la Paw generada por el respirador en un paciente sin esfuerzo ventilatorio espontáneo, está determinado por la suma de 3 presiones, la presión estática, la presión resistiva y la PEEP[9] (Figura 2).

La presión resistiva es la diferencia de presión entre la presión medida por el respirador en la entrada de la vía aérea y la presión alveolar. Dicho delta de presión está determinado por la resistencia y el flujo inspiratorio[10].

$\Delta \mathrm{P}=\operatorname{Rrs} \times \mathrm{Vi}$

Donde $\Delta P$ corresponde a la diferencia de presiones entre la presión pico y la presión meseta de la vía aérea (Delta PicoPlateau), Rrs es la resistencia del sistema respiratorio y Vi es flujo inspiratorio.

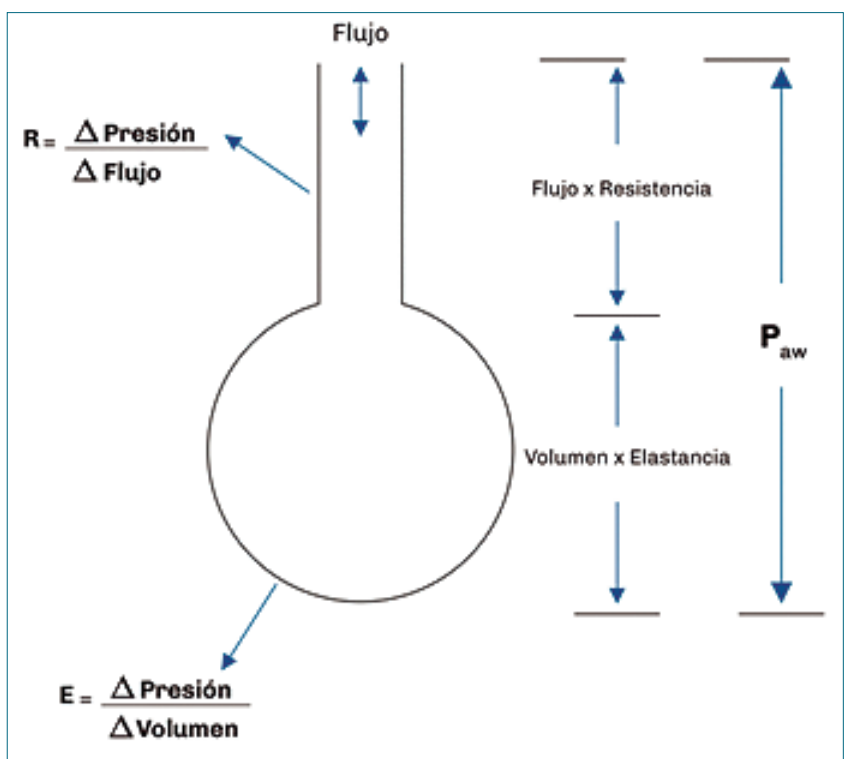

Figura 1. Modelo monocompartimental del sistema respiratorio[6]. Donde $\mathrm{R}$ es resistencia del sistema respiratorio, E es elastancia del sistema respiratorio, Paw es la presión de la vía aérea. Adaptado de Chatburn[7]. 


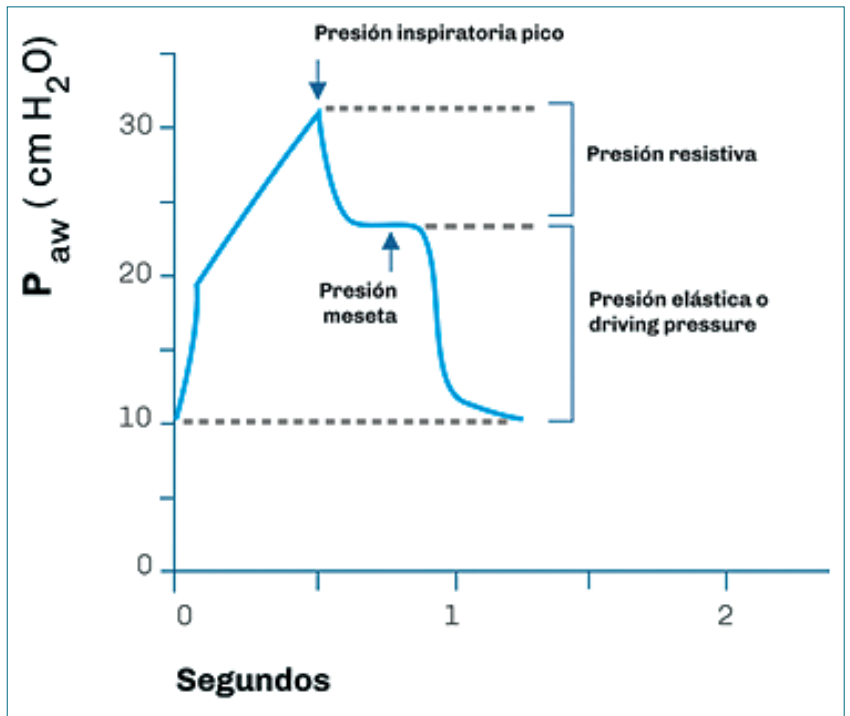

Figura 2. Curva presión-tiempo de VCV. Al comienzo del flujo, se produce un aumento de presión casi vertical (debido a las fuerzas de fricción generadas por el flujo de gas), que es necesario para vencer la resistencia del sistema respiratorio. Luego la forma de la curva cambia a un aumento lineal hasta su valor máximo que es la presión pico[8]. Luego ocurre una caída de presión hasta la presión meseta. Dicha diferencia de presión $\left(\mathrm{P}_{\text {pico }}-\mathrm{P}_{\text {plateau }}\right)$ se corresponde a la presión resistiva y finalmente en la exhalación la presión desciende hasta el valor de PEEP. Dicha diferencia de presión ( $\mathrm{P}_{\text {platea }}$ - PEEP) se corresponde a la presión elástica. Adaptado de Pham[9].

\section{Utilidad práctica}

En Ventilación controlada por Volumen (VCV), la caída de presión desde la presión pico a la presión meseta ( $\Delta$ Pico-Plateau $=\mathrm{P}_{\text {pico }}-\mathrm{P}_{\text {plateau }}$ ) estará dada por la resistencia del sistema y por el flujo inspiratorio[11]. A mayor resistencia del sistema (o, a mayor flujo inspiratorio) mayor será la diferencia entre la $P_{\text {pico }} \mathrm{Y}$ la $P_{\text {plateau }}$

En Ventilación controlada por Presión (PCV), la presencia de una resistencia disipará la presión y el volumen entregado al paciente será menor (para esa misma diferencia de presión). Por ende los aumentos de resistencia en ventilación controlada por presión conllevan el riesgo de disminuir el Vt e hipoventilar al paciente. Sin embargo, si se prolonga el tiempo inspiratorio de forma tal que el flujo inspiratorio llegue a cero, se recupera el mismo Vt entregado a pesar del aumento de la resistencia.

La presión elástica es la diferencia de presión entre la presión al final de la inspiración menos la presión al final de la espiración y está determinada por la elastancia del sistema respiratorio (pulmón y caja torácica) y el volumen corriente[4]. Notablemente este componente de la ecuación del movimiento se corresponde, como veremos, con la airway driving pressure[4].

$\Delta \mathrm{P}=\operatorname{Ers} \times \Delta \mathrm{V}$

Donde $\Delta P$ corresponde a la diferencia de presión entre la presión meseta de la vía aérea y la PEEP (presión positiva al final de la espiración), Ers es la elastancia del sistema respiratorio y $\Delta V$ es el volumen tidal.

\section{Utilidad práctica}

En VCV, considerando la elastancia del sistema respiratorio constante, para un aumento del volumen corriente, mayor será la diferencia de presión generada por el respirador para entregar dicho volumen. Del mismo modo, si aumenta la elastancia del sistema, a Vt constante, también aumentará la presión elástica o la airway driving pressure.

En PCV, el volumen entregado dependerá de la presión inspiratoria programada, de la elastancia del sistema respiratorio, de la resistencia de la vía aérea y del tiempo inspiratorio. Como se mencionó, la resistencia no limitará la entrega de volumen si se prolonga el tiempo inspiratorio lo suficiente de forma tal de llegar a cero.

La ecuación del movimiento constituye además la base teórica para definir los modos ventilatorios de ventilación mandatoria continua controlada por volumen (VCV) y ventilación mandatoria continua controlada por presión (PCV)(6).

En PCV el respirador controla el lado izquierdo de la ecuación, y frente a aumentos de la elastancia o la resistencia, caerá el flujo inspiratorio y por ende el volumen tidal sin modificarse el valor de presión inspiratoria configurada en el respirador[6]:

$\mathrm{PaW}=\Delta \mathrm{E} \times \Delta \mathrm{V}+\Delta \mathrm{R} \times \Delta \mathrm{Vi}$

Donde Paw corresponde a la presión de la via aerea, se halla del lado izquierdo de la ecuación (de color azul) y es la variable controlada por el respirador en PCV.

En VCV el respirador controla el lado derecho de la ecuación, y frente a aumentos de la elastancia o de la resistencia, permanecerá constante el volumen tidal, a expensas de un aumento de la presión pico del respirador[6]:

$\Delta \mathrm{PaW}=\Delta \mathrm{E} \times \mathrm{V}+\Delta \mathrm{R} \times \mathrm{Vi}$

Donde ExV y RxVi se hallan del lado derecho de la ecuación (de color azul) de las cuales las variables volumen o flujo son controlados por el respirador en VCV.

\section{Presión de la vía aérea}

La presión de la vía aérea es muchas veces asumida como el reflejo de las fuerzas aplicadas en el pulmón y es utilizada para monitorizar la ventilación mecánica en la práctica clínica. Esta asunción es errónea porque la presión de la vía aérea es una medida de las propiedades elásticas y resistivas del sistema respiratorio en su totalidad, cuyo comportamiento depende de sus características propias y de la interacción de sus componentes: el pulmón y la caja torácica[12],[13]. Consecuentemente, la presión de la vía aérea, actúa en estas dos estructuras elásticas ubicadas en serie[4].

Por definición, presión es una fuerza aplicada sobre una unidad de superficie.

La presión de la vía aérea, es la presión que distiende todo el sistema respiratorio (pulmón y caja torácica).

$P_{a w}=P_{L}+P_{p l}$

Donde, $P_{a w}=$ presión de la vía aérea (airway pressure) $P_{L}=$ presión transpulmonar $P_{p l}=$ presión pleural o su subrogante, la presión esofágica. 
Hablar vagamente de presión de la vía aérea, es hacer referencia a la suma de las presiones entregadas por el respirador para desplazar al pulmón y a la caja torácica y a la presión requerida para sobreponerse a las fuerzas resistivas (cuando hay flujo presente) que constituyen la presión pico. En ausencia de flujo, y con la vía aérea y el tubo endotraqueal permeables, la presión de la vía aérea medida por el respirador se equilibra con la presión alveolar. Esta presión, llamada presión meseta, es también la requerida para sobreponerse a la elastancia de la caja torácica, la cual por ende, no refleja confiablemente la carga de presión a la cual está expuesta el pulmón en forma aislada[14].

En presión control (PCV), el flujo inspiratorio puede llegar a cero al final del tiempo inspiratorio; si esto ocurre, la presión inspiratoria máxima (PIM) o presión inspiratorio pico (PIP) configurada en el respirador, será igual a la presión Meseta de volumen control[15].

La presión de la vía aérea entonces, es la presión medida al inicio del tubo endotraqueal por el respirador, y dependiendo del momento de la medición puede referirse como presión pico (que incluye los componentes elásticos y resistivos), como presión meseta (asumida como equivalente a la presión alveolar cuando el flujo es cero durante la inspiración) y como la PEEP (si la consideramos a la presión de la vía aérea al final de la espiración) (16). De esto se desprende que se pueden encontrar en la bibliografía menciones por ejemplo a una "airway peak pressure" $\left(P_{\text {aw pico }}\right)$ o una "airway plateau pressure" $\left(P_{\text {aw plateau }}\right)$, presión pico de la vía aérea y presión meseta de la vía aérea respectivamente. Es decir que la denominación general de presión de la vía aérea (Paw), puede referirse a la presión pico, a la presión meseta o a la PEEP ( $\left.P_{\text {aw PEEP }}\right)$. Todas estas presiones involucran las fuerzas requeridas para distender a todo el sistema respiratorio, es decir al pulmón y a la caja torácica (Figura 3).

La $P_{\text {aw plateau }}$ es la presión meseta y está determinada por el $V t$, el volumen que genera la PEEP y la elastancia del sistema respiratorio en pacientes con soporte ventilatorio completo. La presión meseta idealmente debe mantenerse por debajo de 25 $30 \mathrm{CmH}_{2} \mathrm{O}$ [15]. En estos límites de presión, se asume que la elastancia de la caja torácica es normal. Una presión meseta alta en contexto de elastancia aumentada de la caja torácica puede ser segura e incluso necesaria para ventilar a un paciente. Por ejemplo paciente obeso morbido en cirugia con neumoperitoneo[15].

$P_{\text {aw plateau }}=($ Vol. tidal + Vol. $P E E P) \times$ Ers

Donde $P_{\text {aw plateau }}$ es la presión meseta, Vol. Tidal es el volumen corriente, Vol. PEEP es el volumen generado por la PEEP que se calcula como el EELV (volumen pulmonar al final de la espiración) menos la CRF (capacidad residual funcional), y el Ers es la elastancia del sistema respiratorio.

\section{Utilidad práctica}

La presión meseta es considerada un subrogante de la presión alveolar al final de la inspiración. Pero esto no significa que refleje la presión de distensión del pulmón aisladamente (medido efectivamente por la presión transpulmonar). La presión meseta, en cambio, representa la presión de retroceso elástico del sistema respiratorio en su conjunto que comprende tanto al pulmón como a la caja torácica[12]. En casos donde la elastan-

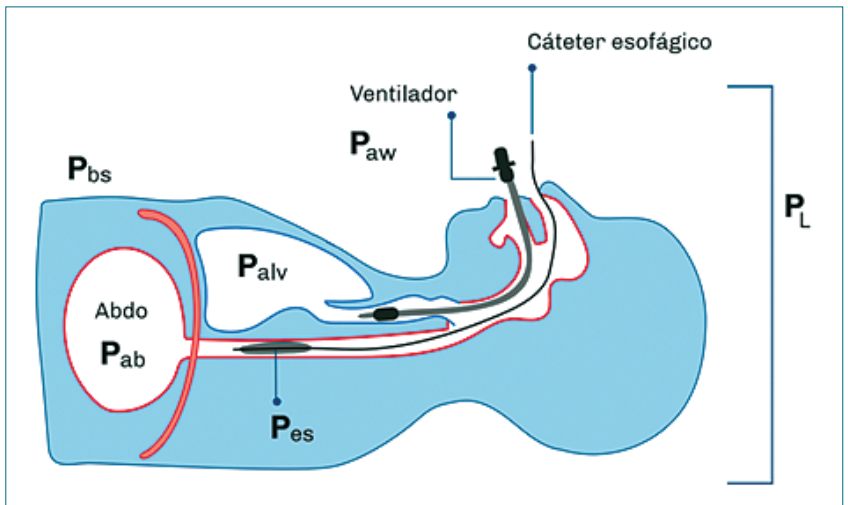

Figura 3. La medición de la Presión de la vía aérea puede realizarse directamente a la entrada de la vía aérea desde el respirador (airway pressure $=\mathrm{P}_{\mathrm{aw}}$ ) y referenciarla con la Presión de la superficie corporal o presión atmosférica (body surface $=\mathrm{P}_{\mathrm{bs}}$ ) la cual por convención es referida con valor de $\mathrm{O}$. La Presión esofágica $\left(\mathrm{P}_{\mathrm{es}}\right)$ puede ser medida con un manómetro esofágico. La presión transpulmonar corresponde al delta de presiones $\left(\mathrm{P}_{\mathrm{L}}=\mathrm{P}_{\text {aw plateau }}-\mathrm{P}_{\text {es }}\right)$. La presión alveolar $\left(\mathrm{P}_{\text {alv }}\right)$ al final de la inspiración puede ser medida desde el respirador como la Paw durante una pausa inspiratoria $\left(P_{\text {aw plateau o meseta }}\right)$. La presión alveolar al final de la espiración también puede ser medida desde el respirador con una pausa espiratoria $\left(\mathrm{P}_{\text {aw PEEp }}\right)$. $\mathrm{P}_{\mathrm{ab}}$ (presión intraabdominal). Adaptado de Henderson[17].

cia de la caja torácica sea normal, el valor de la presión meseta se acercará al valor de la presión transpulmonar. En cambio, si la elastancia de la caja torácica está aumentada, el valor de la presión meseta puede ser alto a expensas de un aumento en la presión pleural, sin que el pulmón esté en riesgo de injuria pulmonar debido a un valor reducido de presión transpulmonar[15],[18]. Otra causa relevante de presión meseta elevada es la alta PEEP (por ejemplo, por limitación del flujo espiratorio e hiperinsuflación dinámica)[19] (Figura 4).

El $\Delta P_{\text {aw }}$ es la airway driving pressure (presión de distensión o de conducción de la vía aérea) y representa la presión por encima de la PEEP aplicada a todo el sistema respiratorio para alcanzar el volumen tidal[20].

Es la oscilación en la presión elástica del sistema respiratorio entre el final de la inspiración y el final de la expiración[17]. Su valor está determinado por el volumen corriente y la elastancia del sistema respiratorio, y describe la relación entre el volumen corriente y el volumen pulmonar disponible para recibirlo[4]. Como mencionamos, este delta de presiones se corresponde con el componente elástico de la ecuación de movimiento:

$\Delta \mathrm{P}_{\mathrm{aw}}=\mathrm{Vt} \times \mathrm{Ers}$

$\Delta \mathrm{P}_{\mathrm{aw}}^{\mathrm{aw}}=\mathrm{Vt} / \mathrm{Crs}$

Donde Volumen tidal o corriente y dado que Crs es la compliancia del sistema respiratorio $=V t /(P . M e s e t a-P E E P)$, entonces:

$\Delta \mathrm{P}_{\mathrm{aw}}=\mathrm{Vt} /(\mathrm{Vt} / \mathrm{P} \cdot$.Meseta-PEEP $)$

$\Delta \mathrm{P}_{\mathrm{aw}}^{\mathrm{aw}}=\forall \mathrm{X} \times(\mathrm{P}$. Meseta-PEEP)/ $\forall t$

$\Delta \mathrm{P}_{\text {aw }}=\mathrm{P} \cdot$.Meseta-PEEP

Es importante reconocer que la $\Delta P_{\text {aw }}$ involucra dos componentes, uno relacionado a la expansión del pulmón y otro rela- 


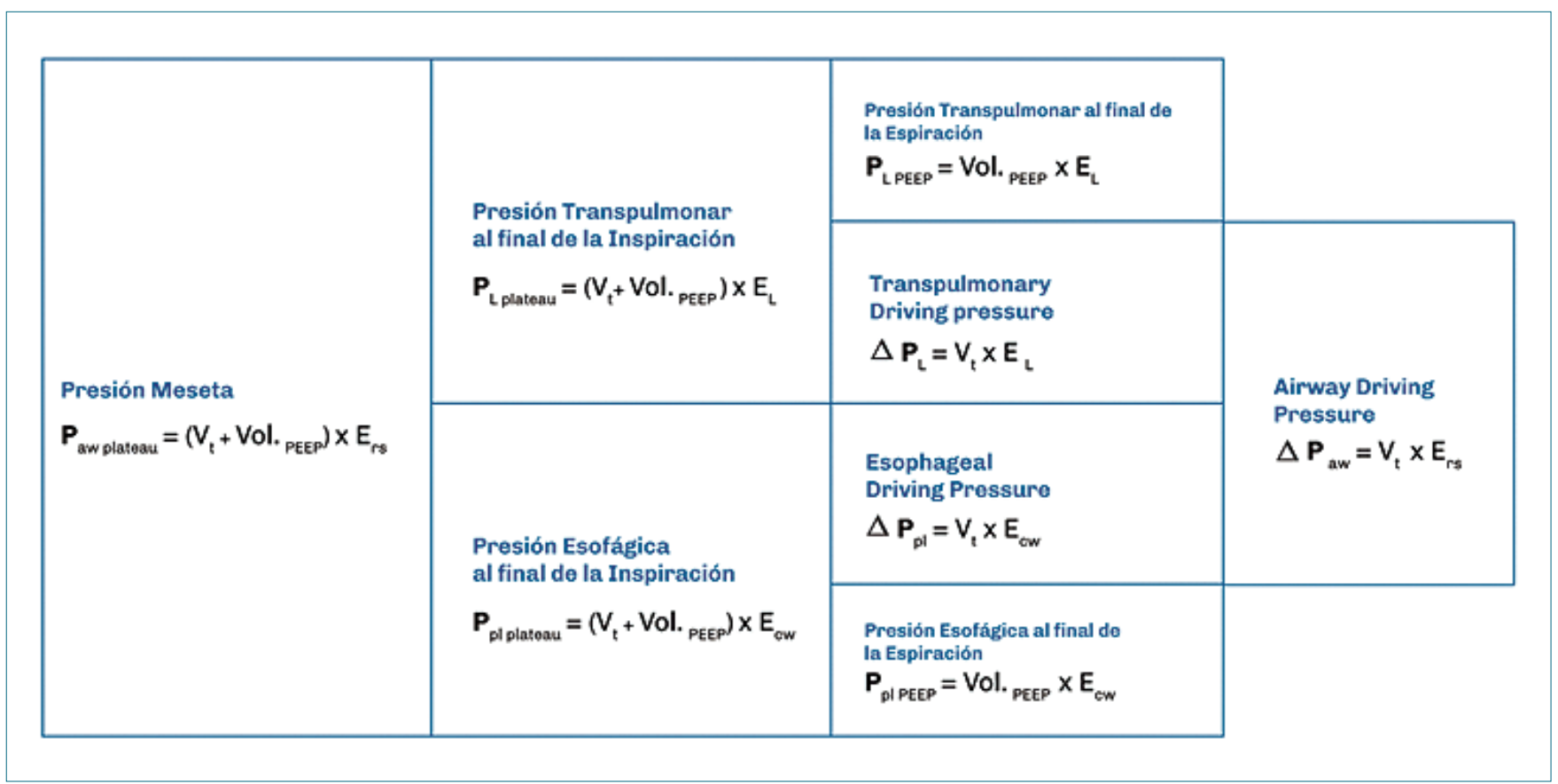

Figura 4. Cuadro con presiones de la vía aérea: determinantes y nomenclatura. Donde Vt es el volumen corriente, $V_{\text {PEEP }}$ es el volumen generado por la PEEP, $E_{r s}$ es la elastancia del sistema respiratorio, $E_{L}$ es la elastancia del pulmón, $E_{c w}$ es la elastancia e la caja torácica, $P_{a w}$ plateau es la presión meseta de la vía aérea, $P_{L \text { plateau }}$ es la presión transpulmonar al final de la inspiración o presión transpulmonar meseta o teleinspiratoria, $P_{\text {es plateau }}$ es la presión esofágica al final de la inspiración o presión esofágica meseta o teleinspiratoria[13], $\Delta \mathrm{P}_{1}$ es la transpulmonary driving pressure o lung driving pressure, $P_{L P E E P}$ es la presión transpulmonar al final de la espiración o presión transpulmonar PEEP o teleespiratoria[13] y representa la fracción de la $P_{L \text { plateau }}$ debido a la PEEP[21], $\Delta \mathrm{P}_{\mathrm{pl}}$ es el cambio en la presión pleural (21) o esophageal driving pressure $\left(\Delta \mathrm{P}_{\mathrm{es}}\right)(27)$ o driving pressure a través de la pared torácica $\left(\Delta \mathrm{P}_{c w}\right)[20], P_{\text {es PEEP }}$ es la presión esofágica al final de la espiración[13] o presión esofágica PEEP o teleespiratoria, la $\Delta \mathrm{P}_{\text {aw }}$ es la airway driving pressure o presión de conducción de la vía aérea. Nota: la suma de $P_{\text {L peEP }}$ y $P_{\text {es peEP }}$ es igual a la PEEP y por tanto, PEEP o $P_{\text {aw PEEP }}=\left(V_{\text {O }} l_{\text {PEEP }} \times E_{r s}\right)$.

cionado a la expansión de la caja torácica[20],[21]. (Ver apartados de transpulmonary driving pressure y esophageal driving pressure).

$\Delta \mathrm{P}_{\text {aw }}=\Delta \mathrm{P}_{\mathrm{L}}$ (transpulmonary driving pressure) $+\Delta \mathrm{P}_{c \mathrm{w}}$ (diferencia de presion pleural o driving pressure a través de la pared torácica o esophageal driving pressure).

\section{Utilidad práctica}

En pacientes con elastancia normal de la caja torácica, la compliancia pulmonar y también la compliancia toracopulmonar se correlacionan directamente con el tamaño del pulmón funcional o aireado (sin áreas de consolidación o colapso) [3],[11],[20]. Dada la relación inversamente proporcional entre la driving pressure y la compliancia toracopulmonar, a mayor pulmón aireado, menor será la driving pressure. Por tanto, tiene más sentido ajustar el Vt buscando la menor driving pressure posible, que configurarla según un valor determinado de peso corporal predicho[22]. De esta manera se logra una mejor adaptación del volumen corriente al pulmón funcional aireado disponible para recibirlo.

Un valor normal de Vt de 6 a $8 \mathrm{~mL} / \mathrm{kg}$ de PBW puede ser excesivo si la driving pressure excede los 13-15 $\mathrm{cmH}_{2} \mathrm{O}[1],[4],[22]$. Por ejemplo, pacientes con SDRA severo con baja compliancia pulmonar, aun la configuración de un $V t$ de $6 \mathrm{ml} / \mathrm{kg}$ puede resultar en alto strain (Vt/CRF)[11]. Y a la inversa, un Vt puede ser mayor a $6 \mathrm{~mL} / \mathrm{kg}$ si la driving pressure se mantiene baja[23] por ejemplo, en un paciente sin patología pulmonar sometido a anestesia general. Cuando se detecta un nivel alto de airway driving pressure, debería aumentarse el reclutamiento pulmonar a través de un nivel de PEEP más elevado para aumentar la compliancia del sistema respiratorio (en pacientes con alta elastancia caja torácica y alta reclutabilidad) o se debería disminuir el Vt (en pacientes con baja elastancia pulmonar y baja reclutabilidad)[1],[17],[18] (Figura 4).

La $P_{L}$ es la presión transpulmonar al final de la inspiración y constituye la presión que distiende solamente el pulmón, y equivale a la diferencia en la presión de la vía aérea medida en condiciones estáticas (presión plateau o meseta) y su correspondiente presión esofágica (presión esofágica al final de la inspiración o presión esofágica meseta o teleinspiratoria)[16]. La presión transpulmonar describe el retroceso elástico a través del pulmón y representa el stress al cual está expuesto el pulmón en cada ventilación[12].

$P_{L}=P_{\text {aw plateau }}-P_{\text {es }}$

Donde, $P_{L}$ es presión transpulmonar al final de la inspiración o presión transpulmonar meseta, $P_{\text {aw plateau }}$ es la presión de la vía aérea al final de la inspiración o presión de la vía aérea meseta y $P_{\text {es }}$ es presión esofágica (como subrogante de presión pleural) al final de la inspiración o presión esofágica meseta. 
La presión pleural $\left(P_{p}\right)$ muchas veces es estimada a través de una catéter esofágico, por eso podría considerarse:

$P_{p l}=P_{\text {es }}$

Donde $P_{\text {es }}=$ presión esofágica

La presión esofágica utilizada como subrogante de la presión pleural, y sus cambios, serán equivalentes a los cambios de ésta última. Los cambios en la presión esofágica durante las presiones pico, plateau y PEEP reflejarán los cambios correspondientes en la presión pleural en esos momentos[16]. La medición de la esofágica permite diferenciar la presión de distensión del pulmón $\left(\mathrm{P}_{\mathrm{L}}\right)$ de la presión disipada a través de la caja torácica[19].

La $P_{L \text { plateau }}$ es proporcional al producto entre la $P_{\text {aw plateau }}$ y la razón de elastancia del pulmón $\left(E_{L}\right)$ sobre la elastancia del sistema respiratorio $\left(E_{r s}\right)(4)$ :

$$
\begin{aligned}
& P_{\text {L plateau }}=P_{\text {aw plateau }} \times\left(E_{L} / E_{r s}\right) \\
& P_{L \text { plateau }}=(V t+V o l P E E P) \times E_{r s} \times\left(E_{L} / E_{r s}\right)
\end{aligned}
$$

Su valor está determinado por el Vt, el volumen que genera la PEEP y la elastancia pulmonar:

$P_{\text {L plateau }}=\left(V t+\right.$ Vol PEEP) $\times E_{L}$

Donde Vol PEEP es la diferencia entre el Volumen pulmonar al final de la espiración (EELV) menos la capacidad residual funcional (CRF).

\section{Utilidad práctica}

La presión transpulmonar nos permite distinguir la presión suministrada al pulmón de la que actúa simplemente para movilizar el abdomen y la caja torácica. Las diferencias entre la presión meseta y la presión transpulmonar meseta son más pronunciadas en obesos y en aquellos con trastornos restrictivos del tórax (causas de elastancia torácica aumentada). Limitar la presión transpulmonar meseta (al final de la inspiración o teleinspiratoria) a menos de $20 \mathrm{cmH}_{2} \mathrm{O}$ parece minimizar el riesgo de distensión alveolar[22],[24] (Figura 4).

La $\Delta P$, es la transpulmonary driving pressure o lung driving pressure y representa la presión de distensión pulmonar, por encima de la PEEP, generada por la entrega del volumen corriente[14]. Y puede calcularse como la diferencia entre la airway driving pressure y la esophageal driving pressure:

$\Delta P_{L}=\Delta P_{\text {aw }}-\Delta P_{p l}$

$\Delta P_{L}=\left(P_{\text {aw }}\right.$ final inspiracion $-P_{\text {aw }}$ final espiración $)-\left(P_{\text {es }}\right.$ final inspiracion- $P$ esinal espiración)

$\Delta \mathrm{P}_{L}=\left(P_{\text {aw plateau }}-P_{\text {aw PEEP }}\right)-\left(P_{\text {es plateau }}-P_{\text {es PEEP }}\right)$

Donde, $\Delta P_{a w}$ es la airway driving pressure, $\Delta P_{p l}$ es la esophageal driving pressure (ver después), $P_{\text {aw }}$ final inspiracion es la presión de la vía aérea medida al final de la inspiración o presión de la vía aérea meseta $\left(P_{\text {aw plateau }}\right), P_{\text {aw }}$ final espiración es la presión de la vía aérea medida al final de la espiración o $P_{\text {aw PEEP }}$ $P$ final de la inspiración es la presión esofágica medida al final de la inspiración o $P_{\text {es plateau }}$ y la $P_{\text {es }}$ final espiración es la presión esofágica medida al final de la espiración o $P_{\text {es PEEP. }}$
La $\Delta P_{L}$ es proporcional al producto entre la $\Delta P_{\text {aw }}$ y la razón de elastancia del pulmón $\left(E_{L}\right)$ sobre la elastancia del sistema respiratorio $\left(\mathrm{E}_{\mathrm{rs}}\right)(25,26)$ (ver después):

$\Delta P_{L}=\Delta P_{\text {aw }} \times\left(E_{L} / E_{s}\right)$

$\Delta P_{L}=\left(V t \times E_{r s}\right) \times\left(E_{L} / E_{r s}\right)$

Su valor está determinado por el volumen corriente y la elastancia pulmonar:

$\Delta P_{L}=V t \times E_{L}$

\section{Utilidad práctica}

Debido a lo engorroso y poco práctico de medir presión esofágica en el ámbito de quirófano, la importancia de este parámetro radica en que su conocimiento y comprensión colabora en la interpretación y uso de los demás parámetros de ventilación protectora. Por ejemplo, en los casos donde la elastancia torácica sea baja, la airway driving pressure, fácil de calcular a la cabecera del paciente, constituirá un adecuado subrogante de la transpulmonary driving pressure[4],[17]. De forma tal que ante pacientes con elastancia no aumentada de la caja torácica, limitar la ventilación a un valor seguro de la airway driving pressure representa un buen objetivo de ventilación protectora (Figura 4).

La $\Delta P_{p l}$ es la esophageal driving pressure o driving pressure a través de la pared torácica y está determinada por el cambio en la presión pleural entre el fin de la inspiración y el fin de la espiración.

$\Delta P_{p l}=\left(P_{\text {es }}\right.$ final de la inspiración o $P_{\text {es plateau }}{ }^{-} P_{\text {es }}$ final de la espiración o $\left.P_{\text {es PEEP }}\right)$

Representa la presión aplicada a la caja torácica por encima de la PEEP al entregarse el volumen tidal. También hallada en la bibliografía como $\Delta P_{c w}$ [20] o $\Delta P_{e s}$ [27]. La $\Delta P_{p /}$ es proporcional al producto entre la $\square$ aw y la razón de elastancia de la caja torácica $\left(E_{c w}\right)$ sobre la elastancia del sistema respiratorio $\left(E_{r s}\right)[25]$ :

$\Delta \mathrm{P}_{p l}=\Delta \mathrm{P}_{a w} \times\left(E_{c w} / E_{r s}\right)$

$\Delta P_{p l}^{p l}=\left(V t \times E_{r s}\right) \times\left(E_{c w}^{c w} / E_{r s}\right)$

Su valor está determinado por el volumen corriente y la elastancia de la caja torácica:

$\Delta P_{p l}=V t \times E_{c w}$

\section{Utilidad práctica}

Representa la presión de distensión de la caja torácica de forma aislada. Su importancia radica en la interpretación de los demás parámetros de presión. Por ejemplo en pacientes con elastancia aumentada de la caja torácica, un valor alto de la airway driving pressure $\left(\Delta P_{a w}\right)$ puede no reflejar un incremento en el riesgo de injuria pulmonar dado que parte importante de dicha presión se corresponde a la esophageal driving pressure $\left(\Delta P_{p l}\right)$ y no a la transpulmonary driving pressure $\left(\Delta P_{L}\right)[15]$.

$\Delta P_{a w}=\Delta P_{p l}+\Delta P_{L}$

$\Delta P_{a w}^{a w}=V t \times E C W+V t \times E L$

Donde $\Delta P_{\text {aw }}$ es airway driving pressure, $\Delta P_{L}$ is transpulmonary 
driving pressure y $\Delta \mathrm{P}_{p l}$ es la driving pressure a traves de la caja toracica o esophageal driving pressure.

Es decir, para cualquier volumen corriente suministrado, a mayor elastancia de la caja torácica $(E C W)$, mayor será la contribución del cambio en la presión pleural $\left(\Delta P_{p}\right)$ al cambio total de la presión de la vía aérea $\left(\Delta P_{a w}\right)$. La caja torácica y el pulmón, contribuyen al valor del cambio de presión de la vía aérea $\left(\Delta P_{a w}\right)$ en proporción a sus elastancias[19] (Figura 4).

\section{Elastancia del sistema respiratorio}

Para discutir las propiedades mecánicas del pulmón y de la caja torácica, y la relación entre éstas, es más conveniente utilizar el concepto de elastancia que el de compliancia[25],[28]. La elastancia es la resistencia a la deformación de un cuerpo frente a una presión aplicada que resulta en un cambio de volumen en dicho cuerpo.

Por su parte, se define a la elastancia del sistema respiratorio como la presión de la vía aérea (presión meseta) necesaria para insuflar a todo el sistema respiratorio en 1 litro $(1.000 \mathrm{~mL})$ por encima de su posición de reposo, es decir, de su CRF (capacidad residual funcional)[13],[25]. Esta presión de la vía aérea, en parte es empleada en inflar el pulmón y en parte es empleada en inflar la caja torácica[28].

La Elastancia del sistema respiratorio $\left(\mathrm{E}_{\mathrm{sr}}\right)$ puede ser calculada de la siguiente manera[13],[25]:

$E_{s r}=\Delta \mathrm{P}_{\text {aw }} / \Delta V$
$E_{s r}=\left(P_{\text {aw plateau }}-P E E P\right) / 1 L$

Donde, $E_{s r}=$ elastancia del sistema respiratorio (respiratory system elastance), $P_{\text {aw plateau }}=$ presión meseta, $P E E P=$ presión positiva al final de la espiración o $P_{\text {aw PEE }} \Delta P_{\text {aw }}=$ airway driving pressure.

Nota: La compliancia es la relación recíproca o inversa de la elastancia $(C=1 / E)$. Describe la facilidad de un cuerpo de ser distendido (29). Se calcula como:

$C_{s r}=\Delta V / \Delta P_{a w}$
$C_{s r}=V t /\left(P_{\text {aw plateau }}-P E E P\right)$

Si bien es un parámetro más familiar dado que sus valores están incorporados en los respiradores, creemos que el desglose de las presiones y demás parámetros de la ventilación protectora son más comprensibles a través del análisis e interpretación de la elastancia. Un valor normal de Crs en paciente en ventilación mecánica debería ser mayor de 50 a $100 \mathrm{ml} / \mathrm{cmH}_{2} \mathrm{O}[29]$.

Esr es entonces la razón entre la presión aplicada al sistema respiratorio y el cambio de volumen que se genera, y se compone de la suma de la elastancia pulmonar o $E_{L}$ (presión necesaria para inflar el pulmón un determinado volumen) y la elastancia de la caja torácica o Ecw (presión requerida para inflar la caja torácica o chest wall al mismo volumen)[13],[25].

Al estar ubicadas ambas estructuras en serie (el pulmón y la caja torácica), la sumatoria de sus elastancias resultará en una elastancia equivalente o total (la del sistema respiratorio) que tendrá un valor mayor al de cada una por separado[29].

Un valor promedio normal de Esr es de alrededor de 16
$\mathrm{CmH}_{2} \mathrm{O} / \mathrm{L}[17]$. La elastancia del sistema respiratorio puede estar disminuida por causas relacionadas con una caja torácica o pulmón poco distensibles o por ambas[29]

$E_{\text {rs }}$ o total $=E_{L}+E_{c w}$

$E_{r s}=\Delta P_{L} / V t+\Delta P_{p /} N t$

$E_{r s}=\left(\Delta P_{a w}-\Delta P_{p}\right) / V t+\Delta P_{p l} / V t$

$E_{r s}=\Delta P_{a w} / V t-\Delta P_{p l} / V t+\Delta P_{p l} / V t$

$E_{r s}^{r s}=\Delta P_{a w}^{a w} N t-\Delta P_{p l}^{p l} N t+\Delta P_{p t}^{p l} N t$

$E_{r s}=\Delta \mathrm{P}_{a w} N t$

$E_{r s}=\left(P_{\text {aw plateau }}-P E E P\right) / V t$

Donde, $E_{s r}=$ elastancia del sistema respiratorio (respiratory system elastance), $E_{L}=$ elastancia del pulmón, $E_{C W}=$ elastancia de la caja torácica, $\Delta P_{L}=$ transpulmonary driving pressure, $\Delta P_{p l}=$ esophageal driving pressure, $\Delta P_{a w}=$ airway driving pressure, $P_{\text {aw plateau }}=$ presión de la vía aérea meseta, $P E E P=$ presión positiva al final de la espiración o $P_{\text {aw PEEP }}$

La Elastancia pulmonar $\left(E_{L}\right)$ es igual a la transpulmonary driving pressure dividido el volumen tidal:

$E_{L}=\Delta P_{L} / V t$

Donde $\Delta P$ es la transpulmonary driving pressure que es igual a $\Delta P_{L}=\Delta P_{a w}-\Delta P_{p /}$

Por lo tanto, la elastancia pulmonar es igual a:

$E_{L}=\left(\Delta P_{a w}-\Delta P_{p}\right) / V t$

Un valor promedio normal de elastancia pulmonar es de alrededor de $9 \mathrm{CmH}_{2} \mathrm{O} / \mathrm{L}[17]$.

Causas de aumento de elastancia pulmonar: intubación selectiva, atrapamiento aéreo e hiperinsuflación dinámica, edema pulmonar, fibrosis pulmonar, SDRA pulmonar, neumonitis, trastornos del tejido conectivo[29].

La Elastancia de la caja torácica $\left(\mathrm{E}_{c w}\right)$ es igual a:

$E_{c w}=\Delta P_{p l} / \Delta V$

Donde $\Delta P_{p l}$ es la esophageal driving pressure que es igual a $\Delta P_{p l}=P_{\text {es plateau }}-P_{\text {es PEEP }}$ $E_{c w}=\left(P_{\text {es plateau }}^{\text {es plateau }}-P_{\text {es PEEP }}^{\text {es PEEP }}\right) / V t$

Donde $P_{\text {es plateau }}$ es la presión esofágica o pleural al final de la inspiración y $P$ es PEEP es la presión esofágica o pleural al final de la espiración.

Un valor promedio normal de elastancia pulmonar es de alrededor de $7 \mathrm{CmH}_{2} \mathrm{O} / \mathrm{L}[17]$.

Causas de aumento de elastancia de la pared torácica: obesidad, ascitis u otros aumentos de la presión intraabdominal por ejemplo neumoperitoneo o embarazo, posición prona, cifoescoliosis, pectus excavatum, enfermedades neuromusculares (Guillain Barre), tumores de la caja torácica, trauma torácico, extensos derrames pleurales[13],[19],[20],[29].

Cuando el volumen del pulmón aumenta, la caja torácica que lo rodea incrementa su volumen en la misma cantidad. Si la pared del tórax es "blanda" (baja elastancia), la presión ge- 
nerada en la interfase entre el pulmón y la caja torácica (la presión pleural o esofágica al final de la inspiración) será baja, por ejemplo de $5 \mathrm{cmH}_{2} \mathrm{O}$. Asumiendo que la presión transpulmonar requerida para inflar un pulmón "rígido" (alta elastancia) es de $25 \mathrm{cmH}_{2} \mathrm{O}$, entonces la presión total aplicada al sistema respiratorio o presión de la vía aérea meseta será de $30 \mathrm{cmH}_{2} \mathrm{O}$ (presión pleural al final de la inspiración de $5 \mathrm{cmH}_{2} \mathrm{O}$ más la presión transpulmonar al final de la inspiración de $25 \mathrm{cmH}_{2} \mathrm{O}$ )[25]. Esta presión meseta de la vía aérea en parte es empleada en inflar el pulmón y en parte es empleada en inflar la caja torácica[28]. En otro caso, en el cual el tórax es poco complaciente (alta elastancia), la presión pleural al final de la inspiración será más alta y por tanto la presión transpulmonar al final de la inspiración será más baja:

$P_{a w}=\uparrow P_{p l}+\downarrow P_{L}$

Donde $P_{a w}$ es presión de la vía aérea meseta, $P_{\text {L }}$ es presión transpulmonar al final de la inspiración o presión transpulmonar meseta, $P_{p l}$ es la presión pleural o esofágica al final de la inspiración o presión esofágica meseta. Como mencionamos, para una determinada presión de la vía aérea, la presión pleural aumenta cuando la elastancia de la caja torácica está elevada y en consecuencia, la presión transpulmonar disminuye[28].

Gattinoni ejemplifica estos conceptos utilizando la razón de elastancias[28]:

Dado que $P_{a w}=P_{L}+P_{p l}$ y que $E_{r s}=E_{L}+E_{c w}$ entonces, $P_{L}=P_{a w} \times\left(E_{L} / E_{S I}\right)$

Donde $P_{L}$ es presión transpulmonar teleinspiratoria o presión transpulmonar meseta, $P_{a w}$ es presión meseta de la vía aérea, $E_{L}$ es elastancia pulmonar y $E_{s r}$ es elastancia del sistema respiratorio.
La presión transpulmonar, el final de la inspiración, es directamente proporcional a la presión meseta de la vía aérea y a la razón de elastancias $\left(E_{L} / E_{r s}\right)$, es decir, a la relación entre la elastancia pulmonar y la elastancia del sistema respiratorio.

En humanos sanos en posición erecta, la relación entre la elastancia del pulmón y la del sistema respiratorio es alrededor de 0,6 (ratio o razón de elastancias), es decir, que el $60 \%$ de la presión de la vía aérea es utilizada para inflar el pulmón y el otro $40 \%$ es usado para expandir la caja torácica[13].

Sin embargo, en pacientes del ámbito de la terapia intensiva, este ratio puede variar de 0,8 (pulmones muy rígidos y caja torácica normal) a 0,2 (pulmones blandos y pared torácica rígida) debido a alteraciones del pulmón o de la caja torácica[13].

Por un lado, para la misma presión aplicada, por ejemplo de $30 \mathrm{cmH}_{2} \mathrm{O}$, y con un ratio de elastancias de 0,8; la presión transpulmonar será de $24 \mathrm{cmH}_{2} \mathrm{O}$ y la presión pleural será de 6 $\mathrm{CmH}_{2} \mathrm{O}$ (Figura 5A).

Mientras que si el ratio fuera de 0,2; la presión transpulmonar sería de solo $6 \mathrm{cmH}_{2} \mathrm{O}$ y la presión pleural de $24 \mathrm{cmH}_{2} \mathrm{O}$ (28) (Figura 5B).

Estos simples cálculos ilustran la importancia de conocer las características mecánicas, tanto, del pulmón como de la caja torácica del paciente que estemos ventilando. La misma presión meseta de la vía aérea puede generar dramáticamente diferentes presiones transpulmonares y pleurales con marcadas consecuencias en la distensión pulmonar (principalmente una función de la presión transpulmonar) y en la hemodinamia (parcialmente una función de la presión pleural)[28].

La presión pleural elevada debido a un aumento de la elastancia de la caja torácica tiene consecuencias a nivel respiratorio y cardiovascular.

A nivel respiratorio, si la presión de vía aérea está representada en su mayoría por un aumento en la presión pleural, im-

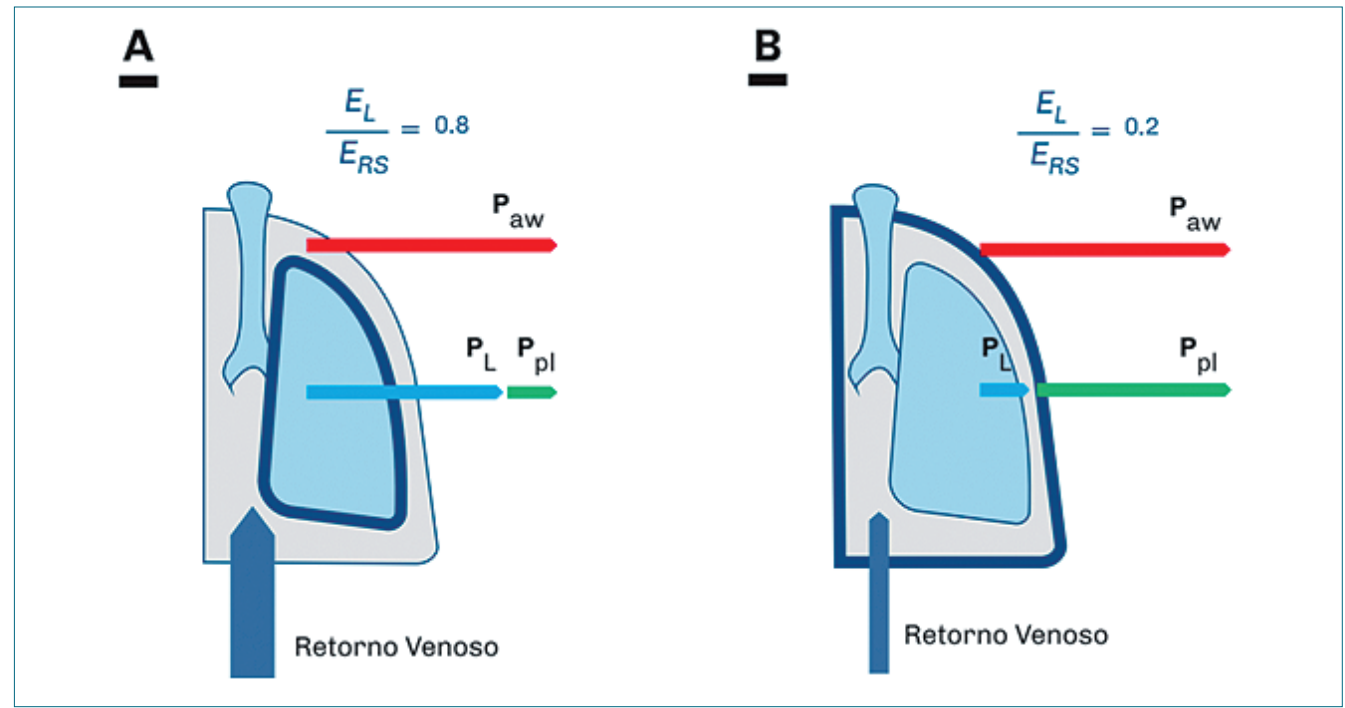

Figura 5. Donde $P_{a w}$ representa la presión meseta de la vía aérea, $P_{\perp}$ representa la presión transpulmonar al final de la inspiración, $P_{p 1}$ representa la presión esofágica al final de la inspiración. A-Paciente con alta elastancia pulmonar. Para la misma presión meseta de la vía aérea, cuando la razón entre la elastancia pulmonar y la elastancia del sistema respiratorio $\left(E_{1} / E_{r s}\right)$ es 0,8 la presión transpulmonar es igual al $80 \%$ de la presión de vía aérea meseta y la presión pleural es igual al 20\% restante; B-Paciente con alta elastancia de la caja torácica. Cuando la razón de elastancias es 0,2 la presión transpulmonar es igual al $20 \%$ de la presión meseta de la vía aérea y la presión pleural es igual al $80 \%$. Para la misma presión de la vía aérea, el retorno venoso depende del incremento en la presión pleural. Por tanto, en este caso en donde la presión pleural representa un alto porcentaje de la presión meseta, el retorno venoso estará disminuido. Adaptado de Gattinoni[13]. 
plica una disminución en la presión transpulmonar, la fuerza de distensión pulmonar. Por lo tanto, una presión transpulmonar disminuida se asocia a una disminución en el strain pulmonar y en el riesgo de generar VILI pero, a su vez, podría ser insuficiente para abrir los alvéolos, generando zonas de atelectasias. En caso de realizarse maniobras de reclutamiento, se requerirá una presión de la vía aérea más alta para alcanzar una presión transpulmonar de apertura suficiente[16],[25], [28].

A nivel hemodinámico, el aumento de la presión pleural puede disminuir el gasto cardíaco al reducir el retorno venoso. Por lo tanto, la evaluación de la hemodinamia, requiere una especial atención en casos de aumentos de la presión intraabdominal. A su vez, la PVC (presión venosa central) y presión de enclavamiento pulmonar pueden aparecer "falsamente" elevadas en presencia de presión pleural aumentada[28],[30].

Ventilar a dos pacientes con la misma presión de la vía aérea (meseta) puede oscilar entre el riesgo de injuria pulmonar si la elastancia del pulmón es alta y la de la caja torácica es baja, a alteraciones hemodinámicas importantes si ocurre la situación inversa[13] (Figura 5A y 5B).

\section{Utilidad práctica}

Está descrito que el ratio de elastancias $\left(E_{L} / E_{S r}\right)$ puede variar en pacientes quirúrgicos desde 0,36 a 0,95 lo cual implica que para una misma presión meseta de la vía aérea por ejemplo de $30 \mathrm{cmH}_{2} \mathrm{O}$, la presión transpulmonar resultante puede ser tan baja como $10 \mathrm{cmH}_{2} \mathrm{O}$ o tan alta como $28 \mathrm{cmH}_{2} \mathrm{O}$ [31]. Incluso, la razón de elastancias puede variar para el mismo paciente en distinto momento de la cirugía, siendo casi de 1 cuando se realiza la inducción anestésica y se paraliza al paciente, o descender a 0,3 en la presencia de neumoperitoneo y trendelemburg[31].

Para una determinada presión aplicada en la vía aérea, cuando la elastancia de la caja torácica esté elevada, la presión pleural va a aumentar. En consecuencia, la presión transpulmonar (la presión de distensión del pulmón) será baja[13],[25]. Esto tiene dos implicancias importantes. Por un lado, en pacientes con alta elastancia de la caja torácica, como ser obesos, embarazadas o en pacientes con otras causas de presión intraabdominal aumentada, se requerirán mayores presiones para una expansión pulmonar adecuada[13]. Y, por lo tanto, estos valores aumentados de presión meseta o airway driving pressure no están implicando riesgo de injuria pulmonar ya que la presión transpulmonar será baja. La segunda implicancia es que la presión pleural elevada repercutirá sobre el retorno venoso y la hemodinamia.

\section{Resistencia del sistema respiratorio}

Durante la inspiración, la presión en la vía aérea es mayor que la presión alveolar y ese gradiente de presión determina el flujo a través de la resistencia inspiratoria. La resistencia inspiratoria total está determinada por la adición en serie de las resistencias del circuito del respirador + las del tubo endotraqueal + la del árbol traqueo bronquial del paciente. Es decir, en las conexiones en serie, las resistencias comparten el mismo flujo pero cada una genera una caída de presión individual, y la resistencia total entonces será la suma de las resistencias individuales arrojando un valor mayor al de cada una de ellas por separado. En cambio, las resistencias de ambos pulmones están vinculadas en paralelo, las cuales actúan como las compliancia en serie, se adicionan recíprocamente. Por ende la resistencia equivalente será menor que cualquiera de las dos individualmente.

La ley enunciada por Luis Marie Poiseuille para condiciones de flujo laminar en 1838 y extendida casi un siglo después (1939) por Heinrich Ludwig Hagen para condiciones de flujo turbulento (Ley de Hagen-Poiseuille), enuncia que la resistencia de un sistema $(R)$ equivale $a[32]$ :

$$
\begin{aligned}
& R=\frac{8 x}{\varpi x} \underline{x} \frac{x}{r^{4}} \text { para condiciones de flujo laminar y } \\
& R=\frac{8 x}{\varpi x} \frac{\mu}{r^{5}} \text { para condiciones de flujo turbulento }
\end{aligned}
$$

Donde $\mu$ es la viscosidad del fluido, I es la longitud del conducto de conducción y r el radio del mismo.

En la medida en que la única variable que usualmente se modifica en el caso del sistema respiratorio es su radio, se constituye prácticamente en el único determinante de la $\mathrm{R} y$, siendo que la misma depende de la $4^{\text {ta }}$ potencia del radio ( $5^{\text {ta }}$ en caso de flujo turbulento), pequeñas modificaciones en este último llevan a grandes cambios en la primera. De esta forma la trascendencia en la práctica clínica de un episodio de broncoespasmo o de una cierta cantidad de moco impactado en el tubo endotraqueal, no deben ser subestimados[32].

Para un valor de $R$ dado, el flujo puede calcularse:

$V i=\Delta P / R$ que puede reescribirse como:

$\Delta P=V i \times R$ lo que constituye el segundo componente de la ecuación del movimiento.

Donde $\Delta P$ es la diferencia de presión o delta de presión, Vi es el flujo inspiratorio y $R$ es la resistencia del sistema respiratorio.

La resistencia inspiratoria se calcula utilizando la diferencia de presión entre la presión pico y la presión meseta y el valor del flujo inspiratorio. Para pacientes intubados y ventilados, la $\mathrm{R}$ debe ser menor a $10 \mathrm{CmH}_{2} \mathrm{O} / \mathrm{L} / \mathrm{s}$. Causas comunes de Raw aumentada son: broncoespasmo, secreciones, diámetro endotraqueal pequeño[15].

\section{Utilidad práctica}

Establecido el flujo inspiratorio, el gradiente de presión generado depende del valor de la Resistencia.

Ej: En VCV, establecido el Vt a entregarse y un determinado tiempo inspiratorio, el gradiente de $\mathrm{P}_{\text {aw pico }}-\mathrm{P}_{\text {aw plateau }}$ generado dependerá del valor de la R. Es decir que frente a aumentos de la resistencia como ser acodamiento del tubo endotraqueal, secreciones, broncoespasmo, etc. la diferencia de presión entre la $\mathrm{P}_{\text {aw pico }}$ y la $\mathrm{P}_{\text {aw plateau }}$ aumentará por incremento en la $\mathrm{P}_{\text {aw pico }}$ [33] $\uparrow \Delta P=V x \uparrow R$

De la misma forma, a un valor de resistencia constante, los cambios en el flujo modifican los gradientes de presión.

Ej: En VCV, establecido el Vt a entregarse, la disminución del tiempo inspiratorio (aumentando la relación l:E de 1:2 a 1:4, aumentando la frecuencia respiratoria o aumentando el tiempo 
de pausa inspiratoria en ciertos respiradores mecánicos), generará un aumento del flujo inspiratorio y eso determinará un aumento en la $\mathrm{P}_{\text {aw pico }} \mathrm{y}$ por ende un aumento en el gradiente de presión entre la $P_{\text {aw pico }}$ y la $P_{\text {aw plateau }}$ sin que esto implique cambios en la resistencia.

$\uparrow \Delta P=\uparrow V \times R$

La resistencia de la vía aérea se puede calcular conociendo datos como la $\mathrm{P}_{\text {aw pico, }}$ la $\mathrm{P}_{\text {aw plateau }}$ y el flujo inspiratorio: $R\left(\mathrm{cmH}_{2} \mathrm{O} / / \times \mathrm{s}\right) \stackrel{\text { aw pico }}{=} P_{\text {aw pico }}-P_{\text {aw plateau }}\left(\mathrm{CmH}_{2} \mathrm{O}\right) /$ flujo inspiratorio (I/S).

La Resistencia total normal en un paciente sano en asistencia respiratoria mecánica es de 10 a $15 \mathrm{cmH}_{2} \mathrm{O} / \mathrm{L} / \mathrm{seg}$.

\section{Stress y Strain}

Los pulmones de pacientes con el mismo tamaño corporal y ventilados con el mismo volumen corriente pueden estar sujetos a diferentes fuerzas dependiendo del tamaño funcional o aireado de sus pulmones. Esto es expresado por los conceptos mecánicos de stress y strain[17].

Cuando una fuerza es aplicada a un cuerpo elástico, este experimentará tensión y se comportará de acuerdo a la Ley de Young[13]:

Stress $=k$. Strain

La fórmula nos muestra que la deformación que experimenta un cuerpo (strain) es proporcional a la tensión a la que es sometido (stress). Aparece también una constante de proporcionalidad (k, o módulo de Young); esta constante es propia del material y da cuenta de su rigidez.

Desde el punto de vista de la física tradicional, el stress es definido como la fuerza reactiva por unidad de área que se desarrolla dentro de un material, en respuesta a la aplicación de una fuerza externa[13],[20]. En mecánica respiratoria, el stress pulmonar refleja las presiones de distensión aplicadas al parénquima pulmonar a la cual se oponen las presiones elásticas generadas por las paredes alveolares y por el andamiaje pulmonar[17]. El stress es entonces, la contrafuerza que se desarrolla dentro de las estructuras del pulmón, como la matriz extracelular, cuando una fuerza de igual intensidad se aplica para estirarla[13]. El stress pulmonar es estimado como la presión transpulmonar (presión de la vía aérea - presión pleural)[34].

Stress $=P_{L}$ (presión transpulmonar)

El strain representa la deformación experimentada por una estructura y es definida como un cambio en longitud o volumen comparado con la longitud o volumen iniciales asumiendo su condición natural sin stress[17],[20]. En mecánica respiratoria, el strain corresponde a la magnitud de deformación del pulmón desde su volumen en reposo cuando se aplica un determinado stress[17].

El strain por tanto, se refiere al cambio de volumen pulmonar (el volumen corriente) relativo a un volumen de referencia (la capacidad residual funcional)[20],[34].

En términos clínicamente relevantes:

Strain $=\Delta V / C R F$

Strain $=V t / C R F$

En presencia de PEEP, el volumen estático incrementado debido a la PEEP debería ser agregado al numerador[13], entonces:

Strain $=(V t+$ volumen de $P E E P) / C R F$

El strain, finalmente, es el incremento del volumen pulmonar que incluye el volumen tidal ("dynamic strain") y el volumen relativo a la PEEP ("static strain") en relación a la CRF[3],[17]. Durante la ventilación, tiene en cuenta ambos componentes, el estático y el dinámico[20]. Bajo la asunción que la Crs refleja la cantidad de pulmón aireado, entonces la airway driving pressure es considerada un subrogante del strain dinámico[1].

El concepto de strain demuestra entonces aún para el mismo peso corporal ideal y el mismo $V t$ pero con diferentes CRF (ej: por posición de trendelemburg), se generan diferentes riesgos de injuria pulmonar[17].

Esta relación (Strain $=V t / C R F)$ demuestra que la reducción del $V t$ disminuye el strain pulmonar, y también que la CRF tiene un efecto en el strain. La CRF marcadamente baja en pacientes con SDRA enfatiza la relevancia de este concepto. Por ejemplo, con un Vt de $500 \mathrm{~mL}$, un pulmón de un paciente sano bajo anestesia general (CRF $2.000 \mathrm{ml}$ ) tendría un strain de 0,25 (500/2.000). Ese mismo Vt en un paciente con SDRA (CRF $500 \mathrm{~mL})$ generaría un strain de 1 (500/500), es decir, 4 veces más strain con el mismo $V t$ por ende aumentando el riesgo de injuria pulmonar[20]. Es decir que limitar el Vt puede no ser suficiente para minimizar el riesgo de injuria pulmonar dado que no considera el tamaño del pulmón aireado (CRF) al cual dicho volumen corriente se aplica[20]. Por lo tanto, el tamaño pulmonar aireado es un factor fundamental a ser considerado para el desarrollo de VILI (Injuria pulmonar inducida por la ventilación mecánica)[16].

El stress y el strain están asociados según:

Stress $=k \times$ Strain es decir,

Barotrauma $=k x$ Volutrauma

$K$ es llamada "elastancia específica"[34]. Representa la pendiente de la curva strain stress (Figura 6). Dado que stress = presión transpulmonar y strain $=\Delta V / C R F$, entonces:

$P_{L}$ (stress) $=E_{L} \times \Delta V / C R F$ (strain)

Donde $E_{L}$ es la Elastancia pulmonar específica[35] y $\Delta V$ es el cambio de volumen por encima de la CRF generado por el Vt y la adición de PEEP, y CRF es la capacidad residual funcional[15].

La elastancia pulmonar específica representa la propiedad intrínseca del pulmón de resistir a una deformación y deriva directamente de su estructura[13]. Cada especie tiene su elastancia pulmonar específica (ej. 12-13 $\mathrm{cmH}_{2} \mathrm{O}$ para el ser humano independientemente de su edad, o talla, o con pulmón sano o distresado y $6 \mathrm{cmH}_{2} \mathrm{O}$ para el cerdo doméstico)[21],[44]. Representa la presión transpulmonar requerida para duplicar la CRF[13],[44]. En otras palabras, $12 \mathrm{cmH}_{2} \mathrm{O}$ es el stress desarrollado en las estructuras pulmonares cuando el volumen de reposo (CRF) es duplicado[35]. Por lo tanto una presión transpulmonar de $13 \mathrm{cmH}_{2} \mathrm{O}$ puede causar que una CRF de $3 \mathrm{~L}$ se infle a $6 \mathrm{~L}$ en una persona normal y que una CRF de 0,5 L se infle hasta $1 \mathrm{~L}$ en un paciente con SDRA[31].

De esta relación arriba mencionada, es evidente que el volutrauma causado por excesivo strain, está estrictamente conectado al barotrauma, causado por excesivo stress, siendo la elastancia específica, la constante de proporcionalidad que los vincula. Por ende, la larga discusión entre los partidarios del 
volutrauma en contra de los partidarios del barotrauma, pierde cualquier significado si nos referimos a la presión transpulmonar en vez de a la presión de la vía aérea, y al strain en vez de al volumen corriente[36]. Por eso mejores subrogantes para hablar del stress pulmonar serían la presión transpulmonar en vez de la presión de la vía aérea meseta $\left(P_{\text {aw plateau }}\right)$ y la airway driving pressure $\left(\Delta P_{a w}\right)$, en vez del $V t$ para hablar del strain pulmonar.

Dado que la elastancia pulmonar específica es constante y representa la pendiente de la curva stress-strain (Figura 6) que permanece lineal hasta su capacidad pulmonar total, el strain puede derivarse del stress y viceversa[13],[21].

El stress podría calcularse a la cabecera del paciente con respiradores que midan CRF[37] y a partir de la elastancia pulmonar específica y calcular el valor de presión transpulmonar sin el requerimiento de un catéter esofágico. A la inversa, estimando la presión transpulmonar a través de un catéter esofágico, podría obtenerse un valor del strain[38].

Como se observa en la Figura 6 cuando la $P$, alcanza 20 $\mathrm{CmH}_{2} \mathrm{O}$, se genera un strain de alrededor 1,5 y parte de los pulmones ingresan en su región de capacidad pulmonar total. Se ha demostrado en animales sanos que cuando la ventilación ocurre en esta área, se desarrolla injuria letal asociada a la ventilación mecánica dentro de las 24 h[21],[39].

\section{Utilidad práctica}

En la práctica clínica, es raro alcanzar estos valores de presión transpulmonar. Sin embargo, en pacientes con distress o en aquellos en donde el pulmón presente también zonas no homogéneas que actúen como stress raisers (-ver más adelante-), el posible efecto es el de duplicarse la presión aplicada localmente. Por ende, una $P_{L}$ (presion transpulmonar) de $10 \mathrm{cmH} 2 \mathrm{O}$, en presencia de stress raisers, puede aumentar regionalmente la $P_{L}$ a $20 \mathrm{CmH}_{2} \mathrm{O}[21]$. De esto se desprende que mientras aguardamos evidencia más definitiva, la $P$, debería ser mantenida posiblemente debajo de los 10 o $12 \mathrm{CmH}_{2} \mathrm{O}$.

Otra implicancia relevante de estos conceptos, es que a través de la utilización de suficiente PEEP, se puede evitar o minimizar tanto el strain dinámico como la sobredistensión[3]. Y, la driving pressure puede ser una herramienta disponible para monitorizar el strain dinámico y el reclutamiento inducido por la PEEP (al aumentar la compliance pulmonar y disminuir la driving pressure)[40].

Como mencionamos entonces, la $P_{\text {aw plateau }}$ y el $V t$ han demostrado ser pobres subrogantes del stress y strain respectivamente. Esto se debe a la alta variabilidad interindividual en la elastancia de la caja torácica para el caso de la presión meseta y de la CRF para el caso del Vt[31]. En cambio, la presión transpulmonar y driving pressure han sido invocados como mejores guías para una ventilación mecánica más segura[2],[31],[41].

\section{Stress Raisers}

El parénquima pulmonar puede ser heterogéneo debido a la presencia de consolidaciones y atelectasias, por ejemplo, en el SDRA. El strain puede ser exacerbado por la presencia de stress amplifiers, interfaces de pulmón aireado y no aireado que amplifican las fuerzas tisulares regionales[17]. Estos límites entre las unidades pulmonares normales y las anormales se han denominado stress raisers, y constituyen regiones donde

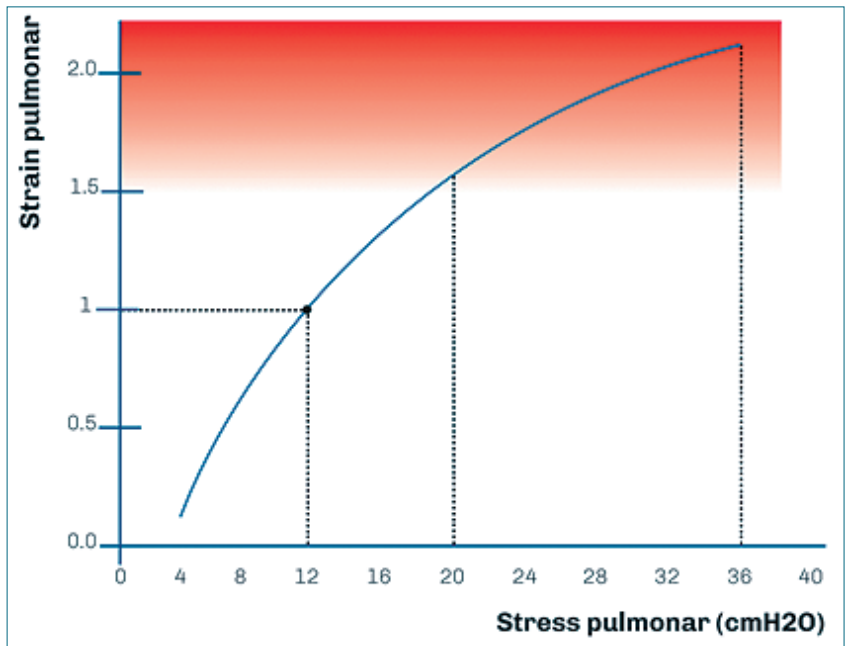

Figura 6. Strain pulmonar (volumen tidal/CRF) como función del stress pulmonar (presión transpulmonar). La pendiente de la curva es la elastancia pulmonar especifica ( $k=$ stress/strain). La duplicación de la Capacidad Residual Funcional ocurre a una presión transpulmonar de 12 $\mathrm{CmH}_{2} \mathrm{O}$ (para una elastancia pulmonar específica de $12 \mathrm{cmH}_{2} \mathrm{O}$ ). Arbitrariamente está indicada una zona anaranjada de "riesgo" de presión transpulmonar, a la cual se corresponde un strain pulmonar que excede el 1,5. Adaptado de Gattinoni[35].

los valores de stress exceden ampliamente los valores de stress globales[17].

El concepto de stress raiser es bien conocido en la ingeniería estructural de los materiales y puede proveer una explicación parcial de por qué, aún ventilando con presiones que son benignas en sujetos sanos, las mismas resulten injuriosas en pacientes con SDRA[17]; y que las intervenciones que promuevan el reclutamiento pulmonar como ser el decúbito prono y la PEEP, puedan disminuir su prevalencia[4] (Figura 7B).

Estos factores locales (stress raisers) pueden multiplicar las presiones aplicadas con el consecuente incremento del stress y strain local. De acuerdo a un modelo teórico desarrollado por Mead en la década de 1970, esto ocurre en mayor medida cuanto mayor sea la falta de homogeneidad pulmonar[16]. Si imaginamos una carga de $5 \mathrm{~kg}$ pendiendo de 5 fibras elásticas, cada fibra soportará $1 \mathrm{~kg}$ de peso. Si por alguna razón (por ejemplo, atelectasias) una de las fibras deja de soportar su fracción de carga, las fibras remanentes deberán soportar ahora $1,25 \mathrm{~kg}$ de peso cada una. Si la heterogeneidad pulmonar se extiende aún más y llega a afectar a 2 fibras, las 3 fibras remanentes soportarán 1,67 kg aproximadamente cada una, y así sucesivamente (Figura 7A).

Cuando se estimó por TAC la mala distribución del strain y el stress en un pulmón patológicamente no homogéneo, se halló un factor de multiplicación de 2 en un cierto porcentaje del pulmón ventilado. Este número representa el factor de multiplicación de la presión transpulmonar en la interface entre dos unidades contiguas de diferente elastancia (debido al colapso de una y a la expansión máxima de la otra). Esto significa que para una ventilación pulmonar con una presión transpulmonar de $15 \mathrm{cmH}_{2} \mathrm{O}$, la presión localmente puede ascender a 30 $\mathrm{CmH}_{2} \mathrm{O}$, con posibles efectos devastadores[36],[42].

Entonces lo que puede considerarse un valor límite seguro de $P$ para el parénquima pulmonar global, en realidad puede 


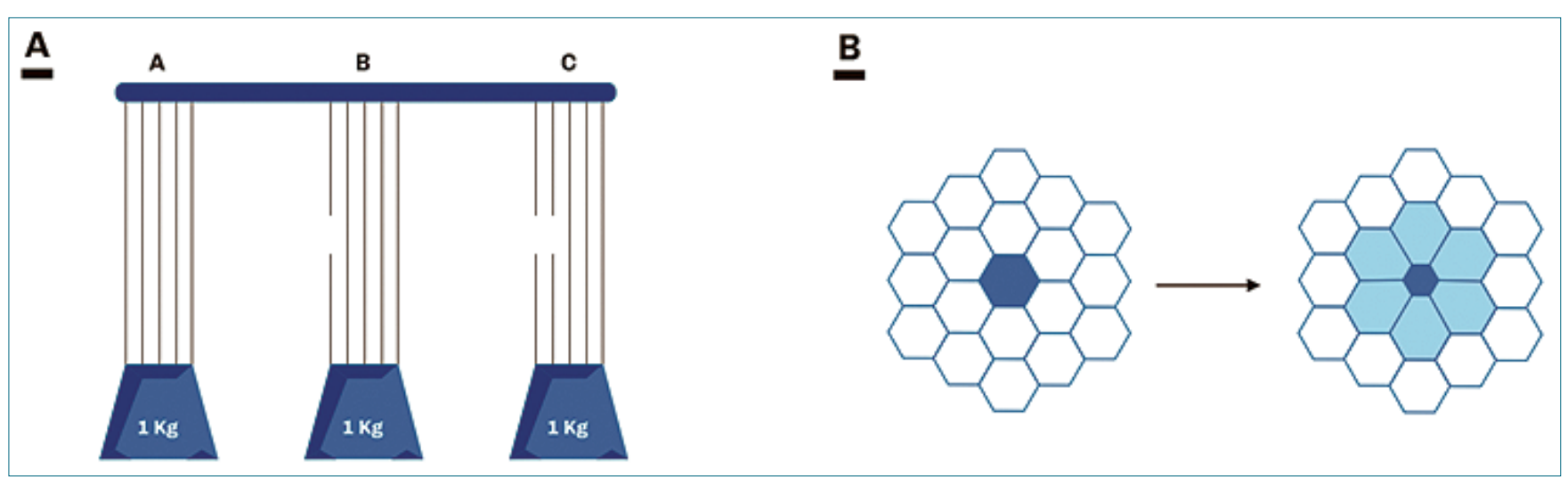

Figura 7. A- Representación gráfica del modelo de Mead. Cuando una carga de $1 \mathrm{k}$ g es impuesta a las 5 fibras elásticas del ejemplo, la carga se distribuye equitativamente entre ellas. Cuando se cuenta con 1 fibra menos, una carga más alta será impuesta a las fibras remanentes (1,25 kg para cada una). En el último caso, las 3 fibras remanentes, cargarán con 1,67 kg cada una. Adaptado de Russotto[4]; B- Un stress raiser es un área local de tejido no homogéneo que multiplica el stress local y el strain en los tejidos que lo rodean. El stress raiser puede ser una región del pulmón colapsada o consolidada que no participa en la ventilación (zona azul oscura de la imagen). Para el mismo volumen pulmonar aplicado en ambos "panales de alveolos", debido a la zona de colapso mencionada, existirán ahora porciones alrededor del stress raiser (zonas grises de la imagen) que estarán sujetas a mayor stress que las normalmente aireadas. La región central colapsada no modifica su tamaño durante la ventilación. Adaptado de Henderson[17].

localmente ejercer un efecto dañino de stress en los límites de las regiones con diferente grado de aireación[4].

\section{Utilidad práctica}

Hasta la fecha, la única intervención disponible para disminuir el número de zonas con stress raisers, ha sido aumentar la homogeneidad de aireación pulmonar[4],[42]; por ejemplo a través del reclutamiento pulmonar por aumento de PEEP y a través del decúbito prono[16],[38]. Esta última puede favorecer una ventilación más homogénea al reducir el fenómeno de apertura y cierre intra-tidal (disminuye la extensión de los stress raisers), especialmente en pacientes con pulmones con alto potencial de reclutabilidad[38].

Los límites de los parámetros de ventilación protectiva que consideramos seguros, pueden no serlo en presencia de zonas no homogéneas pulmonares que actúen como stress raisers. En un pulmón no homogéneo, el cual es usualmente el caso en los pacientes ventilados mecánicamente, la presencia de stress raiser como las atelectasias pueden crear aumentos regionales peligrosos de presión transpulmonar a pesar un valor aceptable de presión meseta y presión transpulmonar[15],[20].

Por tanto, en ausencia de herramientas a la cabecera del paciente para estimar la proporción de amplificadores de stress raisers, los valores de la presión meseta, la driving pressure o la presión transpulmonar deberían ser más bajos que los límites considerados seguros[4],[15],[36].

\section{Stress Index}

Durante VCV, el análisis de la curva de presión-tiempo puede sumar información útil para asistir a la correcta programación del respirador. En condiciones de flujo constante y asumiendo que la compliancia del sistema respiratorio permanece constante, la presión de la vía aérea aumenta linealmente en función del tiempo[1] (Figura 8 B).
La no linealidad en la rama ascendente de la curva presióntiempo durante la inspiración implica que para un intervalo de tiempo dado, el cambio de presión no es constante. Como la entrega de volumen a lo largo del tiempo sí lo es (flujo constante), estos cambios de presión a lo largo del tiempo implicarían cambios en la compliancia, ya que esta última es la propiedad que relaciona cambios de presión y cambios de volumen[1]. A mismos cambios de volumen, pero a diferentes cambios de presión, entonces la compliance del sistema se modifica.

Cuando la compliancia se incrementa durante la inspiración, como ocurre durante un fenómeno de reclutamiento pulmonar intra-tidal, la pendiente de la curva de presión- tiempo disminuye, resultando en una concavidad inferior (Figura 8 A). Por lo tanto, el paciente en el que ocurre el fenómeno de reclutamiento intra-tidal, se podría beneficiar con un aumento de la peep para estabilizar los alvéolos y evitar la apertura y cierre cíclicos de las unidades alveolares, un mecanismo, potencialmente lleva a la injuria pulmonar[1],[19].

Por el otro lado, si la compliancia del sistema respiratorio disminuye durante la inspiración, representado como una concavidad superior en la curva de presión-tiempo, esto sugiere que el pulmón es cíclicamente sobredistendido durante la inspiración (Figura $8 \mathrm{C}$ ). En este caso, una reducción de la PEEP y/o el Vt podría ser beneficioso[1],[17],[19].

Valores del stress index menores a 1 se relacionan a reclutamiento intra-tidal y valores del stress index mayores a 1 se asocian a sobredistensión alveolar cíclica[15],[45]

\section{Utilidad práctica}

En el contexto de ventilación protectiva, el uso del stress index podría ayudar a los anestesiólogos a configurar correctamente el Vt y titular cuidadosamente el nivel de PEEP. La medición del stress index requiere de cálculos complejos que van más allá del objetivo de esta revisión, sin embargo, su estimación cualitativa puede realizarse a simple vista en la curva de presióntiempo de cualquier respirador de quirófano que la posea[1]. 


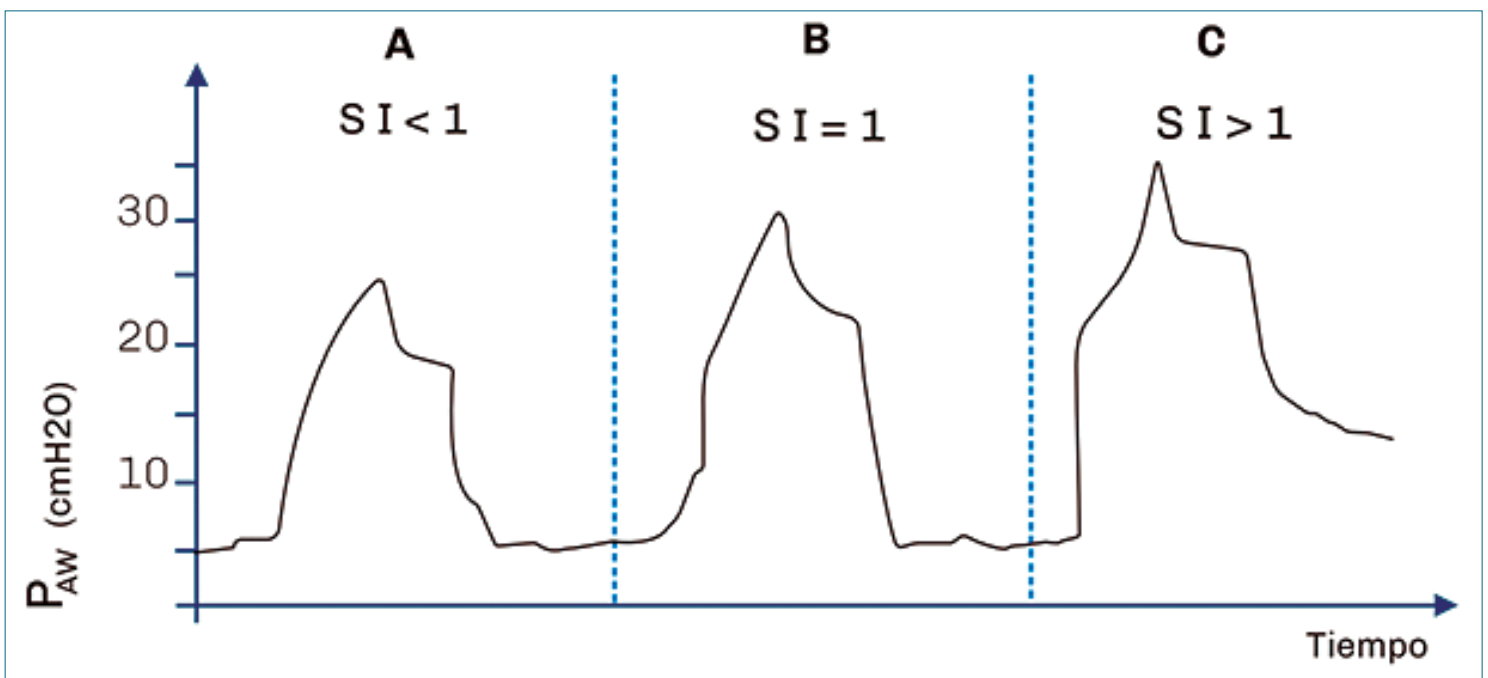

Figura 8. Stress Index. Valores de SI menores a 1 se correlacionan con probable reclutamiento intratidal. SI de 1, mecánica alveolar estable. Valores de SI mayores a 1 se corresponden con probable sobredistensión tidal[43]. Paw = presión de la vía aérea. Adaptado de referencia Henderson[17].

\section{Conclusiones}

La ventilación mecánica es una herramienta que puede salvar la vida de los pacientes, y es requerida en múltiples ocasiones para la realización de intervenciones quirúrgicas. Sin embargo, no está exenta de efectos deletéreos, especialmente a nivel pulmonar. Por ello, es de importancia fundamental el conocimiento de aspectos básicos concernientes a la mecánica del aparato respiratorio. Las presiones de la vía aérea como las presión meseta y la airway driving pressure dentro de límites considerados seguros y protectores, no lo serán tanto, si existen inhomogeneidades pulmonares. A la inversa, presiones meseta o airway driving pressure en valores límites, pueden ser considerados no injuriosos para el pulmón en presencia de elastancia aumentada de la caja torácica. Estos ejemplos demuestran que el mayor conocimiento de la mecánica respiratoria, nos permitirá comprender los efectos de la ventilación mecánica en el pulmón, en la hemodinamia, y nos brindará herramientas para seleccionar una estrategia ventilatoria adecuada para los pacientes

Agradecimientos: A la División de Anestesia, Analgesia y Reanimación del Hospital de Clínicas "José de San Martín" de Buenos Aires por su apoyo. A Maria A. Placenti por su participación en el diseño de las figuras.

\section{Referencias}

1. Ball L, Costantino F, Fiorito M, Amodio S, Pelosi P. Respiratory mechanics during general anaesthesia. Ann Transl Med. 2018 Oct;6(19):379. https://doi.org/10.21037/atm.2018.09.50 PMID:30460253

2. Mietto C, Malbrain ML, Chiumello D. Transpulmonary pressure monitoring during mechanical ventilation: a bench-to-bedside review. Anaesthesiol Intensive Ther. 2015;47(Spec No):s27-37. https://doi.org/10.5603/AIT.a2015.0065 PMID:26575165
3. Silva PL, Pelosi P, Rocco PR. Optimal mechanical ventilation strategies to minimize ventilator-induced lung injury in non-injured and injured lungs. Expert Rev Respir Med. 2016 Dec;10(12):1243-5. https://doi.org/10.1080/17476348.2016.125 1842 PMID:27766893

4. Russotto V, Bellani G, Foti G. Respiratory mechanics in patients with acute respiratory distress syndrome. Ann Transl Med. 2018 Oct;6(19):382. https://doi.org/10.21037/atm.2018.08.32 PMID:30460256

5. Neto AS, Hemmes SN, Barbas CS, Beiderlinden M, FernandezBustamante A, Futier E, et al.; PROVE Network Investigators. Association between driving pressure and development of postoperative pulmonary complications in patients undergoing mechanical ventilation for general anaesthesia: a metaanalysis of individual patient data. Lancet Respir Med. 2016 Apr;4(4):272-80. https://doi.org/10.1016/S2213-2600(16)000576 PMID:26947624

6. Dexter AM, Clark K. Ventilator Graphics: Scalars, Loops, \& Secondary Measures. Respir Care. 2020 Jun;65(6):739-59. https://doi. org/10.4187/respcare.07805 PMID:32457168

7. Chatburn RL. Fundamentals of Mechanical Ventilation. A short course on the theory and application of mechanical ventilators. 1st ed. Cleveland Heights (OH): Mandu Press Ltd.; 2003.

8. Lucangelo $U$, Bernabé $F$, Blanch L. Respiratory mechanics derived from signals in the ventilator circuit. Respir Care. 2005 Jan;50(1):55-65. PMID:15636645

9. Pham T, Brochard LJ, Slutsky AS. Mechanical Ventilation: state of the Art. Mayo Clin Proc. 2017 Sep;92(9):1382-400. https://doi. org/10.1016/j.mayocp.2017.05.004 PMID:28870355

10. Grasselli G, Brioni M, Zanella A. Monitoring respiratory mechanics during assisted ventilation. Curr Opin Crit Care. 2019;1: https://doi.org/10.1097/MCC.0000000000000681 PMID:31738232

11. Silva PL, Rocco PR. The basics of respiratory mechanics: ventilator-derived parameters. Ann Transl Med. 2018 Oct;6(19):376. https://doi.org/10.21037/atm.2018.06.06 PMID:30460250

12. Pham T, Telias I, Beitler JR. Esophageal Manometry. Respir Care. 
2020 Jun;65(6):772-92. https://doi.org/10.4187/respcare.07425 PMID:32457170

13. Gattinoni L, Giosa L, Bonifazi M, Pasticci I, Busana M, Macri $\mathrm{M}$, et al. Targeting transpulmonary pressure to prevent ventilator-induced lung injury. Expert Rev Respir Med. 2019 Aug;13(8):737-46. https://doi.org/10.1080/17476348.2019.163 8767 PMID:31274034

14. Grieco DL, Chen L, Brochard L. Transpulmonary pressure: importance and limits. Ann Transl Med. 2017 Jul;5(14):285. https://doi. org/10.21037/atm.2017.07.22 PMID:28828360

15. Hess DR. Respiratory mechanics in mechanically ventilated patients. Respir Care. 2014 Nov;59(11):1773-94. https://doi. org/10.4187/respcare.03410 PMID:25336536

16. Gattinoni L, Tonetti T, Quintel M. Regional physiology of ARDS. Crit Care. 2017 Dec;21(S3 Suppl 3):312. https://doi.org/10.1186/ s13054-017-1905-9 PMID:29297365

17. Henderson WR, Chen L, Amato MB, Brochard LJ. Fifty Years of Research in ARDS. Respiratory Mechanics in Acute Respiratory Distress Syndrome. Am J Respir Crit Care Med. 2017 Oct;196(7):822-33. https://doi.org/10.1164/rccm.201612-2495Cl PMID:28306327

18. Cruz FF, Ball L, Rocco PR, Pelosi P. Ventilator-induced lung injury during controlled ventilation in patients with acute respiratory distress syndrome: less is probably better. Expert Rev Respir Med. 2018 May;12(5):403-14. https://doi.org/10.1080/17476348.201 8.1457954 PMID:29575957

19. Brochard L, Martin GS, Blanch L, Pelosi P, Belda FJ, Jubran A, et al. Clinical review: respiratory monitoring in the ICU - a consensus of 16. Crit Care. 2012 Dec;16(2):219. https://doi.org/10.1186/ cC11146 PMID:22546221

20. Williams EC, Motta-Ribeiro GC, Vidal Melo MF. Driving Pressure and Transpulmonary Pressure: How Do We Guide Safe Mechanical Ventilation? Anesthesiology. 2019 Jul;131(1):15563. https://doi.org/10.1097/ALN.0000000000002731 PMID:31094753

21. Mauri T, Yoshida T, Bellani G, Goligher EC, Carteaux G, Rittayamai N, et al.; PLeUral pressure working Group (PLUG-Acute Respiratory Failure section of the European Society of Intensive Care Medicine). Esophageal and transpulmonary pressure in the clinical setting: meaning, usefulness and perspectives. Intensive Care Med. 2016 Sep;42(9):1360-73. https://doi.org/10.1007/ s00134-016-4400-x PMID:27334266

22. Rackley CR. Monitoring During Mechanical Ventilation. Respir Care. 2020 Jun;65(6):832-46. https://doi.org/10.4187/respcare.07812 PMID:32457174

23. Nieman GF, Satalin J, Andrews P, Habashi NM, Gatto LA. Lung stress, strain, and energy load: engineering concepts to understand the mechanism of ventilator-induced lung injury (VILI). Intensive Care Med Exp. 2016 Dec;4(1):16. https://doi.org/10.1186/ s40635-016-0090-5 PMID:27316442

24. Beitler J. Lung protection in acute respiratory distress syndrome: what should we target? Curr Opin Crit Care 2019, 25:000-000. https://doi.org/10.1097/MCC.0000000000000692.

25. Gattinoni L, Vagginelli F, Chiumello D, Taccone P, Carlesso E. Physiologic rationale for ventilator setting in acute lung injury/ acute respiratory distress syndrome patients. Crit Care Med. 2003 Apr;31(4 Suppl):S300-4. https://doi.org/10.1097/01. CCM.0000057907.46502.7B PMID:12682456

26. Chiumello D, Carlesso E, Cadringher P, Caironi P, Valenza F, Polli $F$, et al. Lung stress and strain during mechanical ventilation for acute respiratory distress syndrome. Am J Respir Crit Care Med. 2008 Aug;178(4):346-55. https://doi.org/10.1164/rccm.20071015890C PMID: 18451319

27. Pinto E, Santos R, Antunes M, Maia L, Padilha G, Machado J, Carvalho A, Fernandes M...Silva P. Static and Dynamic Transpulmonary Driving Pressures Affect Lung and Diaphragm Injury during Pressure-controlled versus Pressure-support Ventilation in Experimental Mild Lung Injury in Rats. Anesthesiology 2020; 132:00-00. https://doi.org/10.1097/ALN.0000000000003060.

28. Gattinoni L, Chiumello D, Carlesso E, Valenza F. Bench-to-bedside review: chest wall elastance in acute lung injury/acute respiratory distress syndrome patients. Crit Care. 2004 Oct;8(5):350-5. https://doi.org/10.1186/cc2854 PMID:15469597

29. Grinnan DC, Truwit JD. Clinical review: respiratory mechanics in spontaneous and assisted ventilation. Crit Care. 2005 Oct;9(5):472-84. https://doi.org/10.1186/cc3516 PMID:16277736

30. Blanch L, Bernabé F, Lucangelo U. Measurement of air trapping, intrinsic positive end-expiratory pressure, and dynamic hyperinflation in mechanically ventilated patients. Respir Care. 2005 Jan;50(1):110-23. PMID:15636649

31. Chiumello D, Carlesso E, Cadringher P, Caironi P, Valenza F, Polli $F$, et al. Lung stress and strain during mechanical ventilation for acute respiratory distress syndrome. Am J Respir Crit Care Med. 2008 Aug;178(4):346-55. https://doi.org/10.1164/rccm.20071015890C PMID:18451319

32. Murias $\mathrm{G}$. Mecánica respiratoria durante la ventilación a presión positiva. In: Andresen M, Bugedo G, Díaz O, Tomicic V. Ventilación Mecánica. Principios y Práctica Clínica, Editorial Mediterráneo 2010, p.41-56.

33. Lucangelo $U$, Bernabè $F$, Blanch L. Lung mechanics at the bedside: make it simple. Curr Opin Crit Care. 2007 Feb;13(1):6472. https://doi.org/10.1097/MCC.0b013e32801162df PMID:17198051

34. Marini JJ. Evolving concepts for safer ventilation. Crit Care. 2019 Jun;23(S1 Suppl 1):114. https://doi.org/10.1186/s13054-0192406-9 PMID:31200734

35. Gattinoni L, Marini JJ, Collino F, Maiolo G, Rapetti F, Tonetti $T$, et al. The future of mechanical ventilation: lessons from the present and the past. Crit Care. 2017 Jul;21(1):183. https://doi. org/10.1186/s13054-017-1750-x PMID:28701178

36. Tonetti T, Vasques F, Rapetti F, Maiolo G, Collino F, Romitti F, et al. Driving pressure and mechanical power: new targets for VILI prevention. Ann Transl Med. 2017 Jul;5(14):286. https://doi. org/10.21037/atm.2017.07.08 PMID:28828361

37. Blankman P, Hasan D, Bikker IG, Gommers D. Lung stress and strain calculations in mechanically ventilated patients in the intensive care unit. Acta Anaesthesiol Scand. 2016 Jan;60(1):69-78. https://doi.org/10.1111/aas.12589 PMID:26192561

38. Protti A, Votta E, Gattinoni L. Which is the most important strain in the pathogenesis of ventilator-induced lung injury: dynamic or static? Curr Opin Crit Care. 2014 Feb;20(1):33-8. https://doi. org/10.1097/MCC.0000000000000047 PMID:24247615

39. Protti A, Cressoni M, Santini A, Langer T, Mietto C, Febres $D$, et al. Lung stress and strain during mechanical ventilation: any safe threshold? Am J Respir Crit Care Med. 2011 May;183(10):1354-62. https://doi.org/10.1164/rccm.20101017570C PMID:21297069

40. Grieco DL, Russo A, Romanò B, Anzellotti GM, Ciocchetti P, Torrini $F$, et al. Lung volumes, respiratory mechanics and dy- 
namic strain during general anaesthesia. Br J Anaesth. 2018 Nov;121(5):1156-65. https://doi.org/10.1016/j.bja.2018.03.022 PMID:30336861

41. Chiumello D, Carlesso E, Brioni M, Cressoni M. Airway driving pressure and lung stress in ARDS patients. Crit Care. 2016 Aug;20(1):276-86. https://doi.org/10.1186/s13054-016-1446-7 PMID:27545828

42. Kollisch-Singule M, Satalin J, Blair SJ, Andrews PL, Gatto LA, Nieman GF, et al. Mechanical Ventilation Lessons Learned From Alveolar Micromechanics. Front Physiol. 2020 Mar;11:233. https://doi.org/10.3389/fphys.2020.00233 PMID:32265735

43. Correger E, Murias G, Chacon E, Estruga A, Sales B, LopezAguilar J, et al. Interpretación de las curvas del respirador en pacientes con insuficiencia respiratoria aguda. Med Intensiva. 2012 May;36(4):294-306. https://doi.org/10.1016/j.medin.2011.08.005 PMID:22014424

44. Chiumello D, Froio S, Mistraletti G, Formenti P, Bolgiaghi L, Cammaroto A, et al. Gas exchange, specific lung elastance and mechanical power in the early and persistent ARDS. J Crit Care. 2020 Feb;55:42-7. https://doi.org/10.1016/j.jcrc.2019.09.022 PMID:31704618

45. Nieman GF, Satalin J, Andrews P, Aiash H, Habashi NM, Gatto LA. Personalizing mechanical ventilation according to physiologic parameters to stabilize alveoli and minimize ventilator induced lung injury (VILI). Intensive Care Med Exp. 2017 Dec;5(1):8. https://doi.org/10.1186/s40635-017-0121-x PMID:28150228 